\title{
Comparison of the Inhibitory Effect of Gonadotropin Releasing Hormone (GnRH) Agonist, Selective Estrogen Receptor Modulator (SERM), Antiprogesterone on Myoma Cell Proliferation In Vitro
}

\author{
Youn-Jee Chung ${ }^{1}$, Boah Chae', Se-Hyun Kwak' ${ }^{1}$, Jae-Yen Song ${ }^{1}$, Ah-Won Lee ${ }^{2}$, Hyun-Hee Jo' ${ }^{1}$, Young-Ok \\ Lew $^{1}$, Jang-Heub Kim¹, Mee-Ran Kim ${ }^{1 凶}$
}

1. Department of Obstetrics and Gynecology, The Catholic University of Korea, Republic of Korea

2. Department of Hospital Pathology, The Catholic University of Korea, Republic of Korea

$\square$ Corresponding author: Mee-Ran Kim, M.D., Ph.D., Professor. Department of Obstetrics and Gynecology, The Catholic University of Korea, 505 Banpo-Dong, Seocho-Gu, Seoul, 137-040, Korea. Tel : 82-2-2258-6170; Fax : 82-2-595-1549; E-mail: mrkim@catholic.ac.kr

(c) Ivyspring International Publisher. This is an open-access article distributed under the terms of the Creative Commons License (http://creativecommons.org/ licenses/by-nc-nd/3.0/). Reproduction is permitted for personal, noncommercial use, provided that the article is in whole, unmodified, and properly cited.

Received: 2013.09.10; Accepted: 2014.01.09; Published: 2014.01.28

\begin{abstract}
Uterine myomas are the most common gynecologic tumor in women of reproductive age. Treatment options of uterine myomas consist of surgical, medical and interventional therapy such as uterine artery embolization or myolysis. Given that it is the most common type of tumor in women of reproductive age, the treatment of uterine myomas must prioritize uterine conservation. There are several drugs for medical treatment of uterine myoma such as gonadotropin releasing hormone $(\mathrm{GnRH})$ agonist, selective estrogen receptor modulator (SERM) and antiprogesterone. The objective of this study was to compare the effect of GnRH agonist, SERM, and antiprogesterone in the treatment of uterine myomas in vitro. The effect of drugs was evaluated through the cell viability assay in cultured leiomyoma cells, western blot analysis of proliferating cell nuclear antigen (PCNA), and BCL-2 protein expression. As a result, mifepristone single-treated group represents the most significant reduction in myoma cell viability and proliferation. When pretreated with leuprolide acetate, raloxifene shows more significant reduction in myoma cell viability and proliferation than mifepristone. This study suggests one of the possible mechanisms how medications act on uterine myoma, especially at the molecular level.
\end{abstract}

Key words: Leiomyoma, Drug therapy, Gonadotropin-releasing hormone agonist, Raloxifene, Mifepristone

\section{INTRODUCTION}

Uterine myomas are the most common gynecologic tumor in women of reproductive age, with the prevalence from $20 \%$ to $40 \%$ depending on the method of diagnosis. Symptoms associated with these smooth muscle cell tumors include menorrhagia, dysmenorrhea, pelvic pain, pelvic pressure, and infertility. Although uterine myomas are the indication for almost $40 \%$ of hysterectomies, most patients are asymptomatic[1].
Etiology of uterine myomas is still unknown, it is generally agreed that growth of these tumors is affected by concentration of sex hormones, proliferation and apoptosis of myometrial cell, and accumulation of extracellular matrix.

Treatment options of uterine myomas consist of surgical, medical, and interventional therapy such as uterine artery embolization or myolysis. Hysterectomy is a definitive treatment of uterine myomas. 
However, given that it is the most common type of tumor in women of reproductive age, the treatment of uterine myomas must prioritize uterine conservation. Even for postmenopausal women or women with no pregnancy plans, it is advisable to consider medical treatment prior to surgical treatment if patients are appropriate for medical treatment to reduce the risk for complications and to save time and cost for surgery and recovery.

There are several drugs for medical treatment of uterine myoma such as GnRH agonist, SERM and antiprogesterone. It has been demonstrated that $\mathrm{GnRH}$ agonist treatment in patients with uterine myomas reduces tumor size, and suppresses some growth factors that are required for tumor growth [2]. Raloxifene is a SERM which binds to the estrogen receptor and behaves as an estrogen antagonist in some tissues while working as an estrogen agonist in other tissues. In a recent clinical study, raloxifene inhibited the growth of uterine myomas in premenopausal women, and significantly decreased the volume of the uterine myomas in postmenopausal women [3, 4]. Mifepristone, a synthetic steroid with antiprogesterone and antiglucocorticoid activity, is effective in treating uterine myomas resulting in symptom relief and reduction of tumor size[5].

There have been several studies for examining the effect of a single drug, but there have been few studies about comparing the effects of three drugs, especially in vitro. The objective of this study was to compare the effect of GnRH agonist, SERM, and antiprogesterone in the treatment of uterine myomas in vitro.

\section{MATERIALS AND METHODS}

\section{Tissue collection and cell culture}

Five uterine myoma tissues were obtained under patient consent, from women who underwent hysterectomy or myomectomy for medically indicated reasons at Seoul St. Mary's Hospital. Informed consent was obtained from each patient before surgery for the use of uterine tissues for the present study. Approval for use of uterine leiomyoma was granted by the Institutional Review Board of Seoul St. Mary's Hospital. The patients ranged in age from 31 to 56 years, with a mean age of 41.2 years. One patient was a postmenopausal woman. Four patients with regular menstrual cycle underwent surgery during early proliferative phase of the cycle without previous hormone therapy.

Uterine myoma tissues were dissected from the uterus and washed with phosphate-buffered saline (PBS). The tissues were minced into small pieces and digested in $1.5 \mathrm{mg} / \mathrm{ml}$ collagenase IV and $50 \mathrm{mg}$
DNase I at $37^{\circ} \mathrm{C}$ for 3 hours. The leiomyoma cells were collected by centrifugation at 1,200 rpm for 3 minutes and washed 3 times with PBS. Isolated leiomyoma cells were cultured in $100 \mathrm{~mm}^{2}$ culture dishes at $37^{\circ} \mathrm{C}$, $5 \% \mathrm{CO}_{2}$ in a humidified atmosphere in phenol red-free Dulbecco's modified Eagle's medium/F12 (DMEM/F12; Gibco Life Technologies, Grand Island, NY) supplemented with $10 \%$ fetal bovine serum, and $100 \mathrm{U} / \mathrm{ml}$ penicillin. The morphological characteristics were observed daily under inverted microscope with the medium changed every two days. When cells reached saturation, they were isolated with Trypsin-EDTA.

\section{Drug treatment}

In order to see the effects of the three reagents and the impact of GnRH agonist pretreatments on the growth of uterine myoma cells, we conducted two types of experiments. The first set was to see the effects of GnRH agonist, raloxifene (Evista ${ }^{\circledR}$, Sigma-Aldrich, St. Louis, MO), and mifepristone (Sigma-Aldrich, St. Louis, MO) single treatments on cell growth, and the second set was to examine the effect of $\mathrm{GnRH}$ agonist pretreatments. Leuprolide acetate (Leuplin $^{\circledR}$, Takeda Pharmaceutical Company Ltd., Japan) was used as the GnRH agonist substance.

In the first type, the cultured cells were treated with one of three solutions: $10^{-9} \mathrm{M}$ leuprolide acetate for 72 hours [6], 10-9 $\mathrm{M}$ raloxifene for 48 hours [7], or $10^{-7} \mathrm{M}$, mifepristone for 48 hours [8]. In the second set, cultures were first pretreated with $10^{-9} \mathrm{M}$ leuprolide acetate for 72 hours then were exposed to either $10^{-9} \mathrm{M}$ raloxifene or $10^{-7} \mathrm{M}$ mifepristone for 48 hours.

\section{Immunohistochemical assay for estrogen receptor (ER), progesterone receptor (PR) using $\mathbf{2}$ different methods and antibodies}

Immunohistochemical assay was performed to evaluate the expression of estrogen receptor (ER) and progesterone receptor (PR) on cultured uterine leiomyoma cells. Each $4-\mu \mathrm{m}$-thick uterine myoma sample was obtained and processed as below. Each specimen was prepared with the ThinPrep1 Processor (Cytyc, Boxborough, MA) according to the manufacturer's manual. Immunohistochemical staining for ER and PR was done on a ThinPrep slide using the BenchMark XT automated slide processing system (Ventana Medical System, Tucson, AZ, USA). The following rabbit monoclonal antibodies were used for primary antibody: ER (Predilute, SP1, Ventana, USA) PR (Predilute, 1E2, Ventana). We considered ER and PR assays positive, if there are at least $1 \%$ positive tumor neuclei in the ThinPrep slide [9]. 


\section{MTT assay}

After being treated with the corresponding reactants in serum-free DMEM/F12 for 48 and 72 hours in a 96-well tissue culture plate, the data were analyzed at $450 \mathrm{~nm}$ and evaluated with 3-(4,5-dimethylthiazol-2-yl)-2,5-diphenyl-tetrazolium bromide (MTT, Colorimetric assay kit, Chemicon Inc., CA, USA) which is modified from the tetrazolium based colorimetric assay.

\section{Western blot analysis for PCNA and BCL-2}

To extract proteins for western blot analysis, leiomyoma cells were cultivated in $1 \times 10^{6} / \mathrm{ml}$ concentration on $100 \mathrm{~mm}^{2}$ dishes then isolated by centrifugation. Once drug treated, cells were lysed at $4^{\circ} \mathrm{C}$ for 30 minutes in the presence of a lysis buffer. The lysates were subsequently centrifuged at $12,000 \mathrm{rpm}$ for 30 minutes at $4^{\circ} \mathrm{C}$ and the supernatants were collected. Protein content in the supernatants was determined Bio-Rad Protein Assay (Bio-Rad, Philadelphia, PA). Each $50 \mu \mathrm{g}$ aliquots of the extracted protein were boiled for 5 minutes then treated with electrophoresis for 2 hours with $100 \mathrm{~V}$ in $10 \%$ sodium dodecyl sulfate (SDS)/polyacrylamide gel. They were then transferred onto a nitrocellulose paper under $360 \mathrm{~mA}$ for 1 hour and the membrane was eliminated of impurities in 5\% nonfat milk and $0.05 \%$ Tween 20 solution for 1 hour.

The blots were exposed overnight in $4^{\circ} \mathrm{C}$ condition to two types of antibodies: a mouse monoclonal antibody to PCNA (Santa Cruz Biotechnology, Inc. Santa Cruz, CA) and a mouse monoclonal antibody to BCL-2 (Santa Cruz Biotechnology, Inc. Santa Cruz, CA) at dilutions of 1:200. They were washed three times in TBST then exposed to the respective secondary antibody (Santa Cruz Biotechnology, Inc., Santa Cruz, CA) in 1 hour at room temperature before being washed again three times in TBST. The characteristic protein bands were obtained using the ECL Western blotting system (Amersham, Piscataway, NJ).

\section{Statistical analysis}

The uterine myoma cell viability of the control group and the treated groups (raloxifene, mifepristone, leuplin) was statistically analyzed using the multiple comparison method. Samples treated with raloxifene and mifepristone, whether leuplin-pretreated or with no pretreatment, were analyzed using the paired $t$-test.

The data were expressed as the mean \pm SD from at least three independent experiments. Statistical significance was determined using paired $t$-test. A difference with a $p<0.05$ was considered statistically significant.

\section{RESULTS}

\section{Presence of immunoreactive cells for ER, PR in myoma cells}

The ER-, PR-expressions in cultured myoma cells were investigated with immunohistochemistry assay. ER and PR were scored at 5, showing a strong positive reaction. Fig. 1 shows cells stained for ER and PR expressions in the myoma cell.

\section{Effect of leuprolide acetate, raloxifene, and mifepristone on proliferation of myoma cells}

Primarily cultured cells treated with leuprolide acetate, raloxifene, and mifepristone were analyzed in comparison with the control group showing a significant reduction in cell viability (Fig. 2). Myoma cell viability was significantly reduced in leuprolide acetate, raloxifene, and mifepristone single-treated groups, in comparison with control group $(p<0.05)$. Mifepristone treatment showed the most significant reduction in cell viability $(37.1 \pm 3.5 \%)$ followed by leuprolide acetate $(65.7 \pm 3.5 \%)$. Raloxifene had the least reduction in cell viability $(79.6 \pm 2.3 \%)$ compared to the other two drugs $(p<0.05)$.

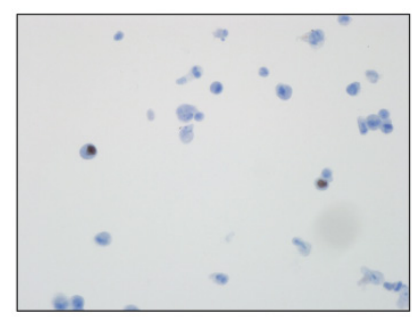

(A)

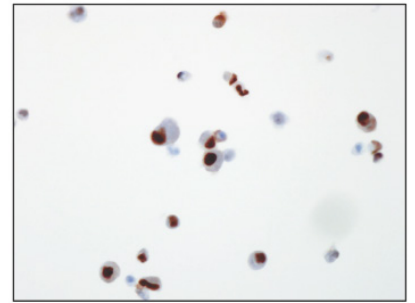

(B)
FIGURE I. Immunohistochemistry of cultured myoma cells; (A) ER-positive myoma cells (B) PR-positive myoma cells. Original magnification: $\times 400$

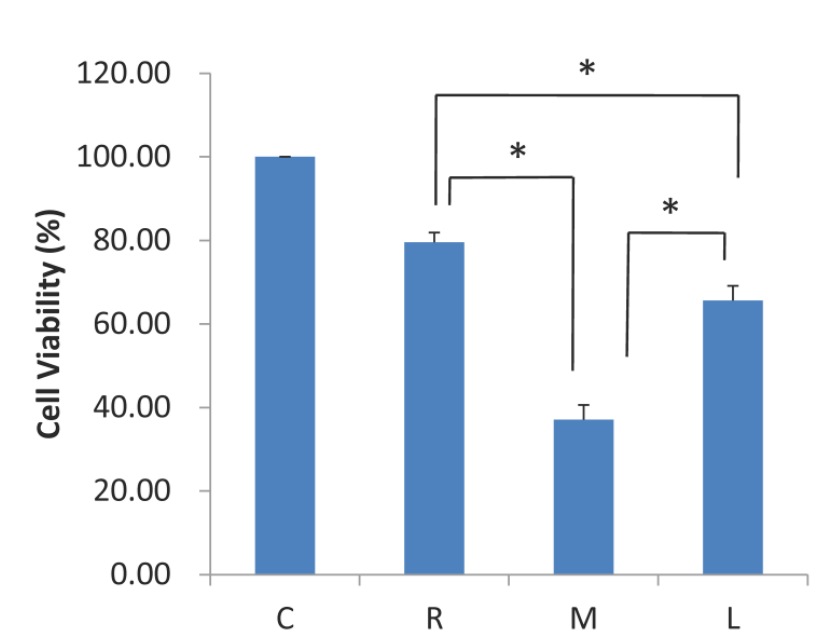

FIGURE 2. Effect of raloxifene, mifepristone and leuprolide acetate on the survival of primarily cultured myoma cells. (C, control; $R$, raloxifene; $M$, mifepristone; L, leuprolide acetate, $* P<0.05$ versus control) 


\section{Effect of raloxifene and mifepristone on the uterine leiomyoma cell proliferation pretreated with leuprolide acetate}

For cells pretreated with $10^{-9} \mathrm{M}$ leuprolide acetate for 72 hours, cell viability was more significantly reduced when treated with raloxifene for 48 hours than for the raloxifene single treatment group $(63.6 \pm 3.5 \%$ versus $79.6 \pm 2.3 \%, p=0.017$ ). However, when they were treated with mifepristone, myoma cell viability was higher than the mifeprostone single treatment group ( $p=0.028$, Fig. 3 ).

\section{Effects of leuprolide acetate, raloxifene, and mifepristone on PCNA and BCL-2 expression}

Western blot analysis of PCNA-positive cells and BCL-2 protein in cultured myoma cells revealed the same result in MTT analysis. In comparison with the control group, PCNA expressions were most significantly reduced in the mifepristone-treated group. In this group, however, the expression increased when pretreated with leupolide acetate (Fig. 4A). For the raloxifene treated group, PCNA expression was reduced when pretreated with leuprolide acetate than when not pretreated.

Experiment with BCL-2 emphasized the results. BCL-2 expression was reduced most significantly in the mifepristone group. However, it was increased with pretreatment with leuprolide acetate. Raloxifene inhibited BCL-2 expression, and with the leuprolide aceate pretreatment, BCL-2 expression was reduced more than its expression in the single treatment group (Fig. 4B).

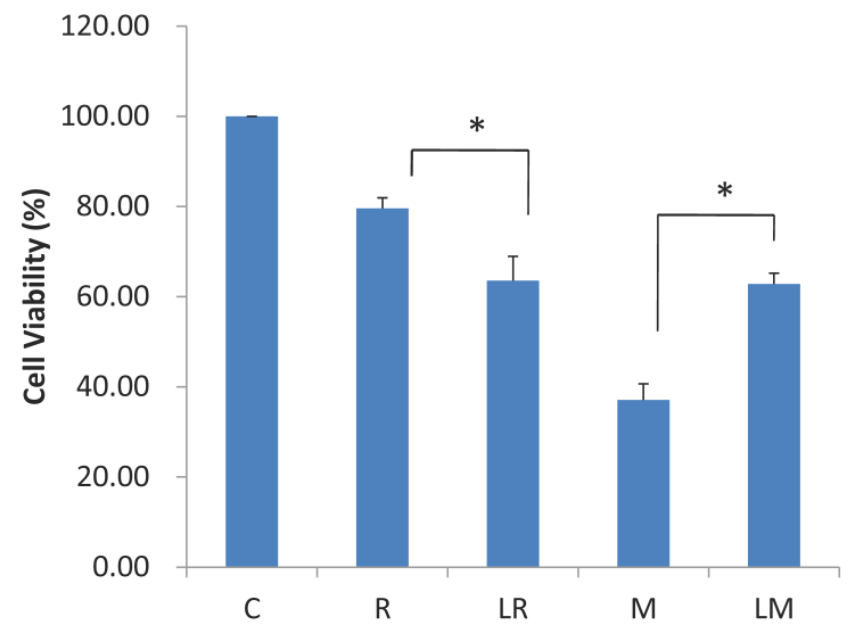

FIGURE 3. Effect of leuprolide acetate on the anti-proliferative effect of raloxifene and mifepristone on primarily cultured myoma cells (C, control; $R$, raloxifene; $L R$, leuprolide acetate + raloxifene; $M$, mifepristone; $L M$, leuprolide acetate + mifepristone, $* P<0.05$ versus control).

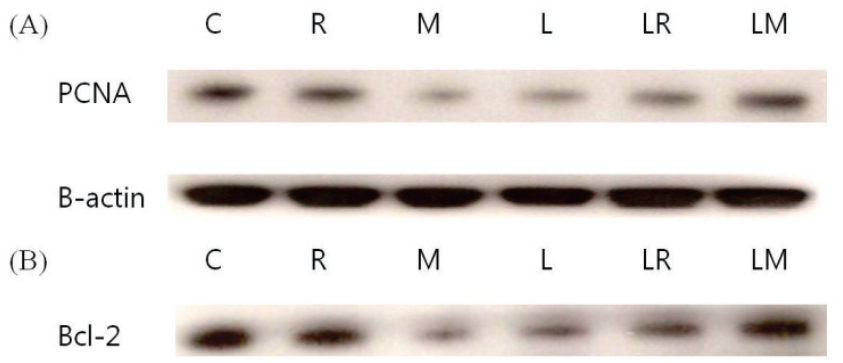

B-actin

FIGURE 4. (A) Effect of raloxifene, mifepristone, and leuprolide acetate on the expression of PCNA on primarily cultured myoma cell and (B) on the expression of BCL-2 on primarily cultured myoma cell. B-actin was used to ensure the even loading of each specimen ( $C$, control; $R$, raloxifene; LR, leuprolide acetate + raloxifene; $M$, mifepristone; LM, leuprolide acetate + mifepristone).

\section{DISCUSSION}

Because uterine myomas usually do not occur before menarche and markedly decrease in size after menopause, it is assumed that growth of uterine myomas depends on estrogen[7]. Several clinical studies substantiated that progestin stimulates growth of uterine myomas, whereas antiprogesterone has the opposite effect [10]. Accumulating data support that sex hormone-lowering medications play an important role in the medical treatment of uterine myomas. The precise mechanism of how medications affect uterine myoma has not yet been elucidated. It is presumed that sex hormones regulate growth of uterine myoma by affecting apoptosis and proliferation of myoma cell.

This study was designed to compare the effect of GnRH agonist, SERM, and antiprogesterone in the treatment of uterine myomas in vitro. In clinical setting, there was no way to compare those antiproliferative effects directly because of the large number of conditions that could not be controlled.

In the present study, the effect of drug was evaluated through MTT assay and western blot analysis of PCNA and BCL-2 protein expression. BCL-2 acts as a biologically important inhibitor of apoptosis. It was discovered that BCL-2 protein expression was rich in uterine myomas than normal myometrium. Abundant expression of BCL-2 protein in uterine myomas may be one of the mechanisms for the growth of uterine myomas[11].

PCNA is a molecule confined to the nucleus of proliferating cells. The determination of PCNA can be useful in the analysis of cell proliferation[12]. As a result, mifepristone single-treated group represents the most significant reduction in myoma cell viability and proliferation. This finding corresponds well with those of the clinical studies which report that mife- 
pristone has been shown to decrease uterine myoma size and menstrual blood loss. When pretreated with leuprolide acetate, raloxifene shows more significant reduction in myoma cell viability and proliferation than mifepristone. This result is similar to the clinical finding in the earlier study.

In postmenopausal women, raloxifene was more effective in treating uterine myomas in premenopausal women due to the markedly decreased levels of circulating estrogen. According to a clinical study, the use of raloxifene appears to be a safe treatment for postmenopausal women affected by uterine myomas in terms of reducing tumor dimensions, incidence of amenorrhea, and the number and severity of abnormal uterine bleeding episodes. Based on these studies, the effect of raloxifene as a medical treatment of uterine myomas will be augmented in the hypoestrogenic status, and is therefore expected to be most effective in treating postmenopausal or elderly premenopausal women. The effectiveness will be further improved if the hypoestrogenic status is maintained simultaneously by treatments such as $\mathrm{GnRH}$ agonist therapy along with raloxifene treatment. This study has also proved that the myoma cell viability of the raloxifene group pretreated with leuprolide acetate was further reduced in comparison to the control group, which is consistent with the conclusions of previous studies. These findings demonstrate the similar results of the previous clinical studies, especially at the molecular level.

Although we could not explain the exact mechanism, there might be a certain role of the GnRH agonist pretreatment that could increase antiproliferative effect of raloxifene. On the other hand, interestingly, $\mathrm{GnRH}$ agonist pretreatment decreased the effect of mifepristone. Further studies will be required to explain this phenomenon.

Studies have suggested that growth of uterine myomas is steroid-dependent and that mitotic activity in uterine myomas is greatest in the luteal phase. The main hormone associated with the luteal phase is progesterone, which is significantly higher during the luteal phase than other phases of the menstrual cycle. Mifepristone, a progesterone receptor modulator with primarily antagonistic properties, has been shown to decrease the size of uterine myomas[13]. The result of this study, in which mifepristone single-treated group represents the most significant reduction in myoma cell viability and proliferation, could explain the effect of progesterone on the growth of uterine myomas in premenopausal women.

It is presumed that medications for treatment of uterine myoma induce apoptosis and inhibit proliferation of uterine myoma. A number of clinical studies showed the effect of drugs from reduced symptoms or decreased uterine myoma size. Few studies on the mechanisms of medical treatment have been found in the literature. There have been few reports on comparing the effects of various kinds of medications and combined therapy. This study suggests one of the possible mechanisms of how medications act on uterine myoma, especially at the molecular level, and compares the effects of medications. In conclusion, mifepristone single-treated group represents the most significant effect on uterine myoma cells in vitro. When pretreated with leuprolide acetate, raloxifene shows more significant effect on uterine myoma than raloxifene monotherapy in vitro. However the antiproliferative effect of mifepristone was decreased with GnRH agonist pretreatment. This study will play a meaningful role as a basic research in elucidating the mechanisms of medical treatment of uterine myomas.

\section{ACKNOWLEDGEMENTS}

This research was supported by Basic Science Research Program through the National Research Foundation of Korea (NRF) funded by the Ministry of Education, Science and Technology (2012R1A1A3020083). The authors thank Chae-Rim Lee for manuscript preparation assistance.

\section{COMPETING INTERESTS}

The authors have declared that no competing interest exists.

\section{REFERENCES}

1. Rackow BW, Arici A. Options for Medical Treatment of Myomas. Obstetrics and Gynecology Clinics of North America. 2006; 33: 97-113. doi:10.1016/j.ogc.2005.12.014.

2. Huang SC. Enhanced Polyadenosine Diphosphate-Ribosylation in Gonadotropin-Releasing Hormone Agonist-Treated Uterine Leiomyoma. Journal of Clinical Endocrinology \& Metabolism. 2003; 88: 5009-16. doi:10.1210/jc.2003-030175.

3. Jirecek S. Raloxifene prevents the growth of uterine leiomyomas in premenopausal women. Fertility and Sterility. 2004; 81: 132-6. doi:10.1016/i.fertnstert.2003.06.009.

4. Palomba S, Sammartino A, Di Carlo C, Affinito P, Zullo F, Nappi C. Effects of raloxifene treatment on uterine leiomyomas in postmenopausal women. Fertil Steril. 2001; 76: 38-43.

5. Bagaria M, Suneja A, Vaid NB, Guleria K, Mishra K. Low-dose mifepristone in treatment of uterine leiomyoma: A randomised double-blind placebo-controlled clinical trial. Australian and New Zealand Journal of Obstetrics and Gynaecology. 2009; 49: 77-83. doi:10.1111/j.1479-828X.2008.00931.x.

6. Wang Y, Matsuo H, Kurachi O, Maruo T. Down-regulation of proliferation and up-regulation of apoptosis by gonadotropin-releasing hormone agonist in cultured uterine leiomyoma cells. European journal of endocrinology / European Federation of Endocrine Societies. 2002; 146: 447-56.

7. Liu J, Matsuo H, Xu Q, Chen W, Wang J, Maruo T. Concentration-dependent effects of a selective estrogen receptor modulator raloxifene on proliferation and apoptosis in human uterine leiomyoma cells cultured in vitro. Human Reproduction. 2007; 22: 1253-9. doi:10.1093/humrep/del515.

8. Luo X, Yin P, Reierstad S, Ishikawa H, Lin Z, Pavone ME, et al. Progesterone and mifepristone regulate L-type amino acid transporter 2 and 4F2 heavy chain expression in uterine leiomyoma cells. The Journal of clinical endocrinology and metabolism. 2009; 94: 4533-9. doi:10.1210/jc.2009-1286.

9. Hammond ME, Hayes DF, Dowsett M, Allred DC, Hagerty KL, Badve S, et al. American Society of Clinical Oncology/College Of American Pathologists guideline recommendations for immunohistochemical testing of estrogen and progesterone receptors in breast cancer. Journal of clinical oncology : official journal of the American Society of Clinical Oncology. 2010; 28: 2784-95. doi:10.1200/jco.2009.25.6529.

10. Chen W. A Novel Selective Progesterone Receptor Modulator Asoprisnil (J867) Inhibits Proliferation and Induces Apoptosis in Cultured Human Uter- 
ine Leiomyoma Cells in the Absence of Comparable Effects on Myometrial Cells. Journal of Clinical Endocrinology \& Metabolism. 2006; 91: 1296-304. doi:10.1210/jc.2005-2379.

11. Baytur YB, Ozbilgin K, Cilaker S, Lacin S, Kurtul O, Oruc S, et al. A comparative study of the effect of raloxifene and gosereline on uterine leiomyoma volume changes and estrogen receptor, progesterone receptor, bcl-2 and p53 expression immunohistochemically in premenopausal women. European Journal of Obstetrics \& Gynecology and Reproductive Biology. 2007; 135: 94-103. doi:10.1016/j.jejogrb.2006.07.042.

12. Palomba S, Orio F, Russo T, Falbo A, Tolino A, Lombardi G, et al. Antiproliferative and proapoptotic effects of raloxifene on uterine leiomyomas in postmenopausal women. Fertility and Sterility. 2005; 84: 154-61. doi:10.1016/j.fertnstert.2004.12.058

13. Steinauer J, Pritts EA, Jackson R, Jacoby AF. Systematic Review of Mifepristone for the Treatment of Uterine Leiomyomata. Obstetrics \& Gynecology. 2004; 103: 1331-6. doi:10.1097/01.AOG.0000127622.63269.8b 